\title{
Some approximation properties of a kind of $q$-Gamma-Stancu operators
}

\section{Chong Zhao ${ }^{1 *}$, Wen-Tao Cheng ${ }^{2}$ and Xiao-Ming Zeng ${ }^{2 *}$}

\footnotetext{
"Correspondence: zhc@xmu.edu.cn; xmzeng@xmu.edu.cn

${ }^{1}$ Department of Computer Science, Xiamen University, Xiamen, 361005, China

${ }^{2}$ Department of Mathematics, Xiamen University, Xiamen, 361005, China
}

\begin{abstract}
In this paper we propose the Stancu type generalization of a kind of $q$-Gamma operators. We estimate the moments of these operators and establish two direct and local approximation theorems of the operators. We also obtain the estimates of the rate of convergence and the weighted approximation of the operators. Furthermore, we present a Voronovskaya type asymptotic formula.
\end{abstract}

MSC: Primary 41A10; 41A25; 41A36

Keywords: q-Gamma-Stancu operators; approximation theorem; weighted approximation; rate of convergence

\section{Introduction}

In 2007, Karsli [1] introduced a kind of new Gamma type operators defined as

$$
L_{n}(f ; x)=\frac{(2 n+3) ! x^{n+3}}{n !(n+2) !} \int_{0}^{\infty} \frac{t^{n}}{(x+t)^{2 n+4}} f(t) \mathrm{d} t, \quad x>0 .
$$

He also estimated the rate of convergence of the operators (1) for functions with derivatives of bounded variation on $(0, \infty)$.

In 2009, Karsli et al. [2] gave an estimate of the rate of pointwise convergence of the operators (1) on a Lebesgue point of a bounded variation function $f$ defined on the interval $(0, \infty)$

In recent years Kim used $q$ calculus to study several results on number theory and the related areas $[3,4]$. Now we mention certain definitions based on $q$-integers, details can be found in [5-7]. For any fixed real number $q>0$ and each nonnegative integer $n$, we denote $q$-integers by $[n]_{q}$, where

$$
[n]_{q}= \begin{cases}\frac{1-q^{n}}{1-q}, & \text { if } q \neq 1 \\ n, & \text { if } q=1\end{cases}
$$

Also $q$-factorial and $q$-binomial coefficients are defined as follows:

$$
\begin{aligned}
& {[n]_{q} != \begin{cases}{[1]_{q}[2]_{q} \cdots[n]_{q},} & \text { if } n=1,2, \ldots ; \\
1, & \text { if } n=0 ;\end{cases} } \\
& {\left[\begin{array}{l}
n \\
k
\end{array}\right]_{q}=\frac{[n]_{q} !}{[n-k]_{q} ![k]_{q} !}, \quad k=0,1, \ldots, n .}
\end{aligned}
$$


It is obvious that $q$-binomial coefficients will reduce to the ordinary case when $q=1$. The $q$-improper integrals are defined as (see $[8,9])$

$$
\int_{0}^{a} f(x) \mathrm{d}_{q} x=(1-q) a \sum_{n=0}^{\infty} f\left(a q^{n}\right) q^{n}, \quad a \in \mathbb{R}
$$

and

$$
\int_{0}^{\infty / A} f(x) \mathrm{d}_{q} x=(1-q) \sum_{-\infty}^{\infty} f\left(\frac{q^{n}}{A}\right) \frac{q^{n}}{A}, \quad A>0,
$$

provided the sums converge absolutely.

The $q$-Beta integral is defined as

$$
B_{q}(t ; s)=K(A ; t) \int_{0}^{\infty / A} \frac{x^{t-1}}{(1+x)_{q}^{t+s}} \mathrm{~d}_{q} x
$$

where $K(x ; t)=\frac{1}{x+1} x^{t}\left(1+\frac{1}{x}\right)_{q}^{t}(1+x)_{q}^{1-t}$ and $(a+b)_{q}^{s}=\prod_{j=0}^{s-1}\left(a+q^{j} b\right), s \in \mathbb{Z}^{+}$.

In particular, for any positive integers $m, n$

$$
K(x ; n)=q^{\frac{n(n-1)}{2}}, \quad K(x ; 0)=1 \quad \text { and } \quad B_{q}(m ; n)=\frac{\Gamma_{q}(m) \Gamma_{q}(n)}{\Gamma_{q}(m+n)},
$$

where $\Gamma_{q}(t)$ is the $q$-Gamma function.

For $m>0$, the $q$-Gamma function is defined by

$$
\Gamma_{q}(m)=\int_{0}^{\frac{1}{1-q}} x^{m-1} E_{q}(-q x) \mathrm{d}_{q} x,
$$

where $E_{q}(x)=\sum_{n=0}^{\infty} q^{\frac{n(n-1)}{2}} \frac{x^{n}}{[n] q !}$. Obviously, it satisfies the following functional equations:

$$
\Gamma_{q}(t+1)=[t]_{q} \Gamma_{q}(t), \quad \Gamma_{q}(1)=1 .
$$

More details of the $q$-Gamma function and the $q$-Beta function can be found in [10].

Very recently, Cai and Zeng [11] proposed a kind of $q$-generalization of Gamma operators and studied their approximation properties. These operators are defined as follows:

$$
G_{n, q}(f ; x)=\frac{[2 n+3]_{q} !\left(q^{n+\frac{3}{2}} x\right)^{n+3} q^{\frac{n(n+1)}{2}}}{[n]_{q} ![n+2]_{q} !} \int_{0}^{\infty / A} \frac{t^{n}}{\left(q^{n+\frac{3}{2}} x+t\right)_{q}^{2 n+4}} f(t) \mathrm{d}_{q} t, \quad x>0 .
$$

In approximation theory the Stancu type modification of operators is an interesting research topic. In this paper, we propose a kind of $q$-Gamma-Stancu operators $G_{n, q}^{\alpha, \beta}(f ; x)$ as follows.

Definition 1 For $f \in C(0, \infty), q \in(0,1), 0 \leq \alpha \leq \beta$ and $n \in \mathbb{N}$, we can define $q$-GammaStancu operators $G_{n, q}^{\alpha, \beta}(f ; x)$ as

$$
G_{n, q}^{\alpha, \beta}(f ; x)=\frac{[2 n+3]_{q} !\left(q^{n+\frac{3}{2}} x\right)^{n+3} q^{\frac{n(n+1)}{2}}}{[n]_{q} ![n+2]_{q} !} \int_{0}^{\infty / A} \frac{t^{n}}{\left(q^{n+\frac{3}{2}} x+t\right)_{q}^{2 n+4}} f\left(\frac{[n]_{q} t+\alpha}{[n]_{q}+\beta}\right) \mathrm{d}_{q} t .
$$


The rest of the paper is organized as follows. In Section 2 we present the moments of the operators $G_{n, q}^{\alpha, \beta}(f ; x)$. In Section 3 we present two direct and local approximation results for the operators $G_{n, q}^{\alpha, \beta}(f ; x)$ by means of the first- and second-order modulus of continuity and the second-order central moment of the operators. In Section 4 we study the rate of convergence of the operators $G_{n, q}^{\alpha, \beta}(f ; x)$. In Section 5 we discuss the weighted approximation theorem and Voronovskaya type asymptotic formula of the operators.

\section{Moment estimates}

In order to obtain the approximation properties of the operators $G_{n, q}^{\alpha, \beta}(f ; x)$, we need the following lemmas.

Lemma 1 ([11]) For any $k \in \mathbb{N}, k \leq n+2$ and $q \in(0,1)$, we have

$$
G_{n, q}\left(t^{k} ; x\right)=\frac{[n+k]_{q} ![n-k+2]_{q} !}{[n]_{q} ![n+2]_{q} !} q^{\frac{2 k-k^{2}}{2}} x^{k}
$$

Lemma 2 If we define the moments as

$$
\begin{aligned}
T_{n, m}^{\alpha, \beta}(x) & =G_{n, q}^{\alpha, \beta}\left(t^{m} ; x\right) \\
& =\frac{[2 n+3] !\left(q^{n+\frac{3}{2}} x\right)^{n+3} q^{\frac{n(n+1)}{2}}}{[n]_{q} ![n+2]_{q} !} \int_{0}^{\infty / A} \frac{t^{n}}{\left(q^{n+\frac{3}{2}} x+t\right)_{q}^{2 n+4}}\left(\frac{[n]_{q} t+\alpha}{[n]_{q}+\beta}\right)^{m} \mathrm{~d}_{q} t
\end{aligned}
$$

then we have

(i) $T_{n, 0}^{\alpha, \beta}(x)=G_{n, q}^{\alpha, \beta}(1 ; x)=1$,

(ii) $T_{n, 1}^{\alpha, \beta}(x)=G_{n, q}^{\alpha, \beta}(t ; x)=\frac{\sqrt{q}[n]_{q}[n+1]_{q}}{\left([n]_{q}+\beta\right)[n+2]_{q}} x+\frac{\alpha}{[n]_{q}+\beta}$, for $n>2$,

(iii) $T_{n, 2}^{\alpha, \beta}(x)=G_{n, q}^{\alpha, \beta}\left(t^{2} ; x\right)=\left(\frac{[n]_{q}}{[n]_{q}+\beta}\right)^{2} x^{2}+\frac{2 \alpha \sqrt{q}[n]_{q}[n+1]_{q}}{\left([n]_{q}+\beta\right)^{2}[n+2]_{q}} x+\left(\frac{\alpha}{[n]_{q}+\beta}\right)^{2}$, for $n>3$.

Proof From Lemma 1, we have $T_{n, 0}^{\alpha, \beta}(x)=G_{n, q}^{\alpha, \beta}(1 ; x)=1$. Thus

$$
\begin{aligned}
T_{n, 1}^{\alpha, \beta}(x) & :=\frac{[2 n+3]_{q} !\left(q^{n+\frac{3}{2}} x\right)^{n+3} q^{\frac{n(n+1)}{2}}}{[n]_{q} ![n+2]_{q} !} \int_{0}^{\infty / A} \frac{t^{n}}{\left(q^{n+\frac{3}{2}} x+t\right)_{q}^{2 n+4}}\left(\frac{[n]_{q} t+\alpha}{[n]_{q}+\beta}\right) \mathrm{d}_{q} t \\
& =\frac{[n]_{q}}{[n]_{q}+\beta} G_{n, q}(t ; x)+\frac{\alpha}{[n]_{q}+\beta} G_{n, q}(1 ; x) \\
& =\frac{\sqrt{q}[n]_{q}[n+1]_{q}}{\left([n]_{q}+\beta\right)[n+2]_{q}} x+\frac{\alpha}{[n]_{q}+\beta} .
\end{aligned}
$$

Finally,

$$
\begin{aligned}
T_{n, 2}^{\alpha, \beta}(x) & :=\frac{[2 n+3]-q !\left(q^{n+\frac{3}{2}} x\right)^{n+3} q^{\frac{n(n+1)}{2}}}{[n]_{q} ![n+2]_{q} !} \int_{0}^{\infty / A} \frac{t^{n}}{\left(q^{n+\frac{3}{2}} x+t\right)_{q}^{2 n+4}}\left(\frac{[n]_{q} t+\alpha}{[n]_{q}+\beta}\right)^{2} \mathrm{~d}_{q} t \\
& =\left(\frac{[n]_{q}}{[n]_{q}+\beta}\right)^{2} G_{n, q}\left(t^{2} ; x\right)+\frac{2[n]_{q} \alpha}{\left([n]_{q}+\beta\right)^{2}} G_{n, q}(t ; x)+\left(\frac{\alpha}{[n]_{q}+\beta}\right)^{2} G_{n, q}(1 ; x) \\
& =\left(\frac{[n]_{q}}{[n]_{q}+\beta}\right)^{2} x^{2}+\frac{2 \alpha \sqrt{q}[n]_{q}[n+1]_{q}}{\left([n]_{q}+\beta\right)^{2}[n+2]_{q}} x+\left(\frac{\alpha}{[n]_{q}+\beta}\right)^{2} .
\end{aligned}
$$


Remark 1 If we put $q=1$ and $\alpha=\beta=0$, we get the moments of the Gamma operators (see [1]):

$$
\begin{aligned}
& L_{n}(t ; x)=\frac{n+1}{n+2} x, \quad n>2, \\
& L_{n}\left(t^{2} ; x\right)=x^{2}, \quad n>3 .
\end{aligned}
$$

Remark 2 Let $n>2$ be a given natural number. For every $q \in(0,1)$ we have

$$
\begin{aligned}
A_{n, q}^{\alpha, \beta}(x)= & G_{n, q}^{\alpha, \beta}(t-x ; x)=\left\{\frac{\sqrt{q}[n]_{q}[n+1]_{q}}{\left([n]_{q}+\beta\right)[n+2]_{q}}-1\right\} x+\frac{\alpha}{[n]_{q}+\beta}, \\
B_{n, q}^{\alpha, \beta}(x)= & G_{n, q}^{\alpha, \beta}\left((t-x)^{2} ; x\right) \\
= & \left(\frac{[n]_{q}}{[n]_{q}+\beta}\right)^{2} x^{2}+\frac{2 \alpha \sqrt{q}[n]_{q}[n+1]_{q}}{\left([n]_{q}+\beta\right)^{2}[n+2]_{q}} x+\left(\frac{\alpha}{[n]_{q}+\beta}\right)^{2} \\
& -2 x\left\{\frac{\sqrt{q}[n]_{q}[n+1]_{q}}{\left([n]_{q}+\beta\right)[n+2]_{q}} x+\frac{\alpha}{[n]_{q}+\beta}\right\}+x^{2} \\
= & \left\{\left(\frac{[n]_{q}}{[n]_{q}+\beta}\right)^{2}-\frac{2 \sqrt{q}[n]_{q}[n+1]_{q}}{\left([n]_{q}+\beta\right)[n+2]_{q}}+1\right\} x^{2} \\
& +2 \alpha\left\{\frac{\sqrt{q}[n]_{q}[n+1]_{q}}{\left([n]_{q}+\beta\right)^{2}[n+2]_{q}}-\frac{1}{[n]_{q}+\beta}\right\} x+\left(\frac{\alpha}{[n]_{q}+\beta}\right)^{2}, \\
C_{n, q}^{\alpha, \beta}(x)= & G_{n, q}^{\alpha, \beta}\left((t-x)^{4} ; x\right) \\
= & \left\{\frac{\left([n]_{q}\right)^{3}[n+3]_{q}[n+4]_{q}}{[n-1]_{q}\left([n]_{q}+\beta\right)^{4} q^{4}}-\frac{4 \sqrt{q}\left([n]_{q}\right)^{2}[n+3]_{q}}{q^{2}\left([n]_{q}+\beta\right)^{3}}\right. \\
& \left.+\frac{6\left([n]_{q}\right)^{2}}{\left([n]_{q}+\beta\right)^{2}}-\frac{4 \sqrt{q}[n]_{q}[n+1]_{q}}{[n+2]_{q}\left([n]_{q}+\beta\right)}+1\right\} x^{4} \\
& +\frac{4 \alpha}{[n]_{q}+\beta}\left\{\frac{\sqrt{q}\left([n]_{q}\right)^{2}[n+3]_{q}}{q^{2}\left([n]_{q}+\beta\right)^{3}}-\frac{3\left([n]_{q}\right)^{2}}{\left([n]_{q}+\beta\right)^{2}}+\frac{3 \sqrt{q}[n+1]_{q}[n]_{q}}{[n]_{q}\left([n]_{q}+\beta\right)}-1\right\} x^{3} \\
& +\frac{6 \alpha^{2}}{\left([n]_{q}+\beta\right)^{2}}\left\{\frac{\left([n]_{q}\right)^{2}}{\left([n]_{q}+\beta\right)^{2}}-\frac{2 \sqrt{q}[n]_{q}[n+1]_{q}}{[n+2]_{q}\left([n]_{q}+\beta\right)}+1\right\} x^{2} \\
& \frac{4 \alpha^{3}}{\left([n]_{q}+\beta\right)^{3}}\left\{\frac{\sqrt{q}[n]_{q}[n+1]_{q}}{[n+2]_{q}\left([n]_{q}+\beta\right)}-1\right\} x+\frac{\alpha^{4}}{\left([n]_{q}+\beta\right)^{4}} .
\end{aligned}
$$

\section{Local approximation}

In this section we establish two direct and the local approximation theorems of the operators $G_{n, q}^{\alpha, \beta}(f ; x)$.

Let $C_{B}[0,+\infty)$ denote the space of all real valued continuous bounded functions $f$ defined on the interval $[0,+\infty)$. The norm $\|\cdot\|$ on the space $C_{B}[0,+\infty)$ is given by $\|f\|=$ $\sup \{|f(x)|: x \in[0,+\infty)\}$.

Further let us consider Peetre's $K$-functional:

$$
K_{2}(f ; \delta)=\inf _{g \in W^{2}}\left\{\|f-g\|+\delta\left\|g^{\prime \prime}\right\|\right\},
$$

where $\delta>0$ and $W^{2}=\left\{g \in C_{B}[0,+\infty): g^{\prime}, g^{\prime \prime} \in C_{B}[0,+\infty)\right\}$. 
For $f \in C_{B}[0,+\infty)$, the modulus of continuity of second order is defined by

$$
\omega_{2}(f ; \delta):=\sup _{0<h \leq \delta} \sup _{x \in[0,+\infty)}|f(x+2 h)-2 f(x+h)+f(x)| .
$$

From $[12,13]$, there exists an absolute constant $M_{0}>0$ such that

$$
K_{2}(f ; \delta) \leq M_{0} \omega_{2}(f ; \sqrt{\delta}), \quad \delta>0 .
$$

Also we set

$$
\omega(f ; \delta):=\sup _{0<h \leq \delta} \sup _{x \in[0,+\infty)}|f(x+h)-f(x)| .
$$

In order to prove the theorems of this section, we need the following lemma.

Lemma 3 Let $q \in(0,1), x \in(0,+\infty), f \in C_{B}[0,+\infty)$. Then, for all $g \in C_{B}^{2}[0,+\infty)$, we have

$$
\left|\hat{G}_{n, q}^{\alpha, \beta}(g ; x)-g(x)\right| \leq\left(B_{n, q}^{\alpha, \beta}(x)+D_{n, q}^{2}(x)\right)\left\|g^{\prime \prime}\right\|,
$$

where

$$
\begin{aligned}
& \hat{G}_{n, q}^{\alpha, \beta}(f ; x)=G_{n, q}^{\alpha, \beta}(f ; x)+f(x)-f\left(T_{n, 1}^{\alpha, \beta}(x)\right), \\
& D_{n, q}^{\alpha, \beta}(x)=T_{n, 1}^{\alpha, \beta}(x)-x .
\end{aligned}
$$

Proof From (15) and Lemma 2, we have

$$
\hat{G}_{n, q}^{\alpha, \beta}(t-x ; x)=0 .
$$

For $x \in(0,+\infty)$ and $g \in C_{B}^{2}[0,+\infty)$, using Taylor's formula,

$$
g(t)-g(x)=(t-x) g^{\prime}(x)+\int_{x}^{t}(t-u) g^{\prime \prime}(u) \mathrm{d} u
$$

we have

$$
\begin{aligned}
\hat{G}_{n, q}^{\alpha, \beta}(g ; x)-g(x)= & \hat{G}_{n, q}^{\alpha, \beta}\left((t-x) g^{\prime}(x) ; x\right)+\hat{G}_{n, q}^{\alpha, \beta}\left(\int_{x}^{t}(t-u) g^{\prime \prime}(u) \mathrm{d} u ; x\right) \\
= & g^{\prime}(x) \hat{G}_{n, q}^{\alpha, \beta}((t-x) ; x)+G_{n, q}^{\alpha, \beta}\left(\int_{x}^{t}(t-u) g^{\prime \prime}(u) \mathrm{d} u ; x\right) \\
& -\int_{x}^{T_{n, 1}^{\alpha, \beta}(x)}\left(T_{n, 1}^{\alpha, \beta}(x)-u\right) g^{\prime \prime}(u) \mathrm{d} u \\
= & G_{n, q}^{\alpha, \beta}\left(\int_{x}^{t}(t-u) g^{\prime \prime}(u) \mathrm{d} u ; x\right)-\int_{x}^{T_{n, 1}^{\alpha, \beta}(x)}\left(T_{n, 1}^{\alpha, \beta}(x)-u\right) g^{\prime \prime}(u) \mathrm{d} u .
\end{aligned}
$$

On the other hand, from

$$
\left|\int_{x}^{t}(t-u) g^{\prime \prime}(u) \mathrm{d} u\right| \leq\left|\int_{x}^{t}\right| t-u|| g^{\prime \prime}(u)|\mathrm{d} u| \leq\left\|g^{\prime \prime}\right\|\left|\int_{x}^{t}\right| t-u|\mathrm{~d} u| \leq(t-x)^{2}\left\|g^{\prime \prime}\right\|
$$


and

$$
\left|\int_{x}^{T_{n, 1}^{\alpha, \beta}(x)}\left(T_{n, 1}^{\alpha, \beta}(x)-u\right) g^{\prime \prime}(u) \mathrm{d} u\right| \leq\left(T_{n, 1}^{\alpha, \beta}(x)-x\right)^{2}\left\|g^{\prime \prime}\right\|=\left(D_{n, q}^{\alpha, \beta}(x)\right)^{2}\left\|g^{\prime \prime}\right\|,
$$

we conclude that

$$
\begin{aligned}
\left|\hat{G}_{n, q}^{\alpha, \beta}(g ; x)-g(x)\right| & =\left|G_{n, q}^{\alpha, \beta}\left(\int_{x}^{t}(t-u) g^{\prime \prime}(u) \mathrm{d} u ; x\right)-\int_{x}^{T_{n, 1}^{\alpha, \beta}(x)}\left(T_{n, 1}^{\alpha, \beta}(x)-u\right) g^{\prime \prime}(u) \mathrm{d} u\right| \\
& \leq G_{n, q}^{\alpha, \beta}\left((t-x)^{2}\left\|g^{\prime \prime}\right\| ; x\right)+\left(D_{n, q}^{\alpha, \beta}(x)\right)^{2}\left\|g^{\prime \prime}\right\| \\
& \leq\left(B_{n, q}^{\alpha, \beta}(x)+\left(D_{n, q}^{\alpha, \beta}(x)\right)^{2}\right)\left\|g^{\prime \prime}\right\| .
\end{aligned}
$$

This completes the proof.

Theorem 1 Let $q \in(0,1), f \in C_{B}[0,+\infty)$. Then, for every $x \in(0,+\infty)$, there exists a constant $M_{1}>0$ such that

$$
\left|G_{n, q}^{\alpha, \beta}(f ; x)-f(x)\right| \leq M_{1} \omega_{2}\left(f ; \sqrt{B_{n, q}^{\alpha, \beta}(x)+\left(D_{n, q}^{\alpha, \beta}(x)\right)^{2}}\right)+\omega\left(f ;\left|D_{n, q}^{\alpha, \beta}(x)\right|\right) .
$$

Proof By (15), we have

$$
\left|\hat{G}_{n, q}^{\alpha, \beta}(f ; x)\right| \leq\left|G_{n, q}^{\alpha, \beta}(f ; x)\right|+2\|f\| \leq 3\|f\| .
$$

Using Lemma 3, for every $g \in C_{B}^{2}[0,+\infty)$, we obtain

$$
\begin{aligned}
\left|G_{n, q}^{\alpha, \beta}(f ; x)-f(x)\right| \leq & \left|\hat{G}_{n, q}^{\alpha, \beta}(f ; x)-f(x)\right|+\left|f(x)-f\left(T_{n, 1}^{\alpha, \beta}(x)\right)\right| \\
\leq & \left|\hat{G}_{n, q}^{\alpha, \beta}(f-g ; x)-(f-g)(x)\right|+\left|f(x)-f\left(T_{n, 1}^{\alpha, \beta}(x)\right)\right| \\
& +\left|\hat{G}_{n, q}^{\alpha, \beta}(g ; x)-g(x)\right| \\
\leq & 4\|f-g\|+\left|f(x)-f\left(T_{n, 1}^{\alpha, \beta}(x)\right)\right|+\left(B_{n, q}^{\alpha, \beta}(x)+\left(D_{n, q}^{\alpha, \beta}(x)\right)^{2}\right)\left\|g^{\prime \prime}\right\| \\
\leq & 4\|f-g\|+\omega\left(f ;\left|D_{n, q}^{\alpha, \beta}(x)\right|\right)+\left(B_{n, q}^{\alpha, \beta}(x)+\left(D_{n, q}^{\alpha, \beta}(x)\right)^{2}\right)\left\|g^{\prime \prime}\right\| .
\end{aligned}
$$

Now, by taking infimum on the right-hand side for all $g \in C_{B}^{2}[0, \infty)$ and using (14), we get the following result:

$$
\begin{aligned}
\left|G_{n, q}^{\alpha, \beta}(f ; x)-f(x)\right| & \leq 4 K_{2}\left(f ; B_{n, q}^{\alpha, \beta}(x)+\left(D_{n, q}^{\alpha, \beta}(x)\right)^{2}\right)+\omega\left(f ;\left|D_{n, q}^{\alpha, \beta}(x)\right|\right) \\
& \leq 4 M_{0} \omega_{2}\left(f ; \sqrt{B_{n, q}^{\alpha, \beta}(x)+\left(D_{n, q}^{\alpha, \beta}(x)\right)^{2}}\right)+\omega\left(f ;\left|D_{n, q}^{\alpha, \beta}(x)\right|\right) \\
& =M_{1} \omega_{2}\left(f ; \sqrt{B_{n, q}^{\alpha, \beta}(x)+\left(D_{n, q}^{\alpha, \beta}(x)\right)^{2}}\right)+\omega\left(f ;\left|D_{n, q}^{\alpha, \beta}(x)\right|\right) .
\end{aligned}
$$

This completes the proof.

Theorem 2 Let $0<\gamma \leq 1$ and $E$ be any bounded subset of the interval $[0,+\infty)$. If $f \in$ $C_{B}[0,+\infty) \cap \operatorname{Lip}_{M_{3}}(\gamma)$, then we have

$$
\left|G_{n, q}^{\alpha, \beta}(f ; x)-f(x)\right| \leq M_{3}\left\{\left(B_{n, q}^{\alpha, \beta}(x)\right)^{\frac{\gamma}{2}}+2(d(x ; E))^{\gamma}\right\}
$$


where $M_{3}$ is a constant depending only on $\alpha, d(x ; E)$ is the distance between $x$ and E defined as

$$
d(x ; E)=\inf \{|t-x|: t \in E \text { and } x \in(0,+\infty)\} .
$$

Proof From the properties of the infimum, there is at least one point $t_{0}$ in the closure of $E$ such that

$$
d(x ; E)=\left|t_{0}-x\right| .
$$

By the triangle inequality, we have

$$
|f(t)-f(x)| \leq\left|f(t)-f\left(t_{0}\right)\right|+\left|f\left(t_{0}\right)-f(x)\right| .
$$

Thus

$$
\begin{aligned}
\left|G_{n, q}^{\alpha, \beta}(f ; x)-f(x)\right| & \leq G_{n, q}^{\alpha, \beta}(|f(t)-f(x)| ; x) \\
& \leq G_{n, q}^{\alpha, \beta}\left(\left|f(t)-f\left(t_{0}\right)\right| ; x\right)+G_{n, q}^{\alpha, \beta}\left(\left|f\left(t_{0}\right)-f(x)\right| ; x\right) \\
& \leq M_{3}\left\{G_{n, q}^{\alpha, \beta}\left(\left|t-t_{0}\right|^{\gamma} ; x\right)+\left|t_{0}-x\right|^{\gamma}\right\} \\
& \leq M_{3}\left\{G_{n, q}^{\alpha, \beta}\left(|t-x|^{\gamma} ; x\right)+2\left|t_{0}-x\right|^{\gamma}\right\}
\end{aligned}
$$

holds. Now we choose $p_{1}=\frac{2}{\gamma}$ and $p_{2}=\frac{2}{2-\gamma}$ such that $\frac{1}{p_{1}}+\frac{1}{p_{2}}=1$, then by Hölder inequality we have

$$
\begin{aligned}
\left|G_{n, q}^{\alpha, \beta}(f ; x)-f(x)\right| & \leq M_{3}\left\{\left[G_{n, q}^{\alpha, \beta}\left(|t-x|^{\gamma p_{1}} ; x\right)\right]^{\frac{1}{p_{1}}}\left[G_{n, q}^{\alpha, \beta}\left(1^{p_{2}} ; x\right)\right]^{\frac{1}{p_{2}}}+2\left|t_{0}-x\right|^{\gamma}\right\} \\
& =M_{3}\left\{G_{n, q}^{\alpha, \beta}\left(|t-x|^{2} ; x\right)^{\frac{\gamma}{2}}+2\left|t_{0}-x\right|^{\gamma}\right\} \\
& =M_{3}\left\{\left(B_{n, q}^{\alpha, \beta}(x)\right)^{\frac{\gamma}{2}}+2(d(x ; E))^{\gamma}\right\} .
\end{aligned}
$$

This completes the proof.

\section{Rate of convergence}

Let $B_{x^{2}}[0,+\infty)$ be the set of all functions $f$ defined on $[0,+\infty)$ satisfying the condition $|f(x)| \leq M_{f}\left(1+x^{2}\right)$, where $M_{f}$ is a constant depending only on $f$. Let $C_{x^{2}}[0,+\infty)$ denote the subset of all continuous functions belonging to $B_{x^{2}}[0,+\infty)$. If $f \in C_{x^{2}}[0,+\infty)$ and $\lim _{x \rightarrow+\infty} \frac{f(x)}{1+x^{2}}$ exists, we write $f \in C_{x^{2}}^{*}[0,+\infty)$. The norm on $C_{x^{2}}^{*}[0,+\infty)$ is given by $\|f\|_{x^{2}}=\sup _{x \in[0,+\infty)} \frac{|f(x)|}{1+x^{2}}$. The modulus of continuity of $f$ on the closed interval $[0, a]$ is defined by

$$
\omega_{a}(f ; \delta)=\sup _{|t-x|<\delta, \delta, t \in[0, a]} \sup _{x \in}|f(t)-f(x)| .
$$

We know that for a function $f \in C_{x^{2}}[0,+\infty)$, the modulus of continuity $\omega_{a}(f ; \delta)$ tends to zero as $\delta \rightarrow 0$.

Now we give a rate of convergence theorem for the operators $G_{n, q}^{\alpha, \beta}(f ; x)$. 
Theorem 3 Let $f \in C_{x^{2}}[0,+\infty), q \in(0,1)$ and let $\omega_{a+1}(f ; \delta)$ be modulus of the continuity of $f$ on the finite interval $[0, a+1] \subset[0,+\infty)$, where $a>0$. Then for $n>3$,

$$
\left|G_{n, q}^{\alpha, \beta}(f ; x)-f(x)\right| \leq 5 M_{f}\left(1+a^{2}\right) B_{n, q}^{\alpha, \beta}(x)+\omega_{a+1}(f ; \delta)\left(1+\frac{1}{\delta}\left[B_{n, q}^{\alpha, \beta}(x)\right]^{\frac{1}{2}}\right) .
$$

Proof For $x \in(0, a]$ and $t>a+1$, since $t-x>1$, we have

$$
\begin{aligned}
|f(t)-f(x)| & \leq M_{f}\left(2+x^{2}+t^{2}\right) \\
& \leq M_{f}\left(2+x^{2}+t^{2}+(2 x-t)^{2}\right) \\
& \leq M_{f}\left(2+3 x^{2}+2(x-t)^{2}\right) \\
& \leq 5 M_{f}\left(1+a^{2}\right)(t-x)^{2} .
\end{aligned}
$$

For $x \in(0, a]$ and $t \leq a+1$, we have

$$
|f(t)-f(x)| \leq \omega_{a+1}(f ;|t-x|) \leq\left(1+\frac{|t-x|}{\delta}\right) \omega_{a+1}(f ;|t-x|)
$$

with $\delta>0$.

From (18) and (19) we get

$$
|f(t)-f(x)| \leq 5 M_{f}\left(1+a^{2}\right)(t-x)^{2}+\left(1+\frac{|t-x|}{\delta}\right) \omega_{a+1}(f ; \delta),
$$

for $x \in(0, a]$ and $t>0$. Thus

$$
\begin{aligned}
\left|G_{n, q}^{\alpha, \beta}(f ; x)-f(x)\right| \leq & G_{n, q}^{\alpha, \beta}(|f(t)-f(x)| ; x) \\
\leq & 5 M_{f}\left(1+a^{2}\right) G_{n, q}^{\alpha, \beta}\left((t-x)^{2} ; x\right) \\
& +\omega_{a+1}(f ; \delta)\left(1+\frac{1}{\delta}\left[G_{n, q}^{\alpha, \beta}\left((t-x)^{2} ; x\right)\right]^{\frac{1}{2}}\right) \\
\leq & 5 M_{f}\left(1+a^{2}\right) B_{n, q}^{\alpha, \beta}(x)+\omega_{a+1}(f ; \delta)\left(1+\frac{1}{\delta}\left[B_{n, q}^{\alpha, \beta}(x)\right]^{\frac{1}{2}}\right) .
\end{aligned}
$$

The proof is completed.

As is well known, if $f$ is not uniformly continuous on the interval $[0, \infty)$, then the usual first modulus of continuity $\omega(f ; \delta)$ does not tend to zero as $\delta \rightarrow 0$. For every $f \in C_{x^{2}}^{*}[0, \infty)$, we would like to take a weighted modulus of continuity $\Omega(f ; \delta)$ which tends to zero as $\delta \rightarrow 0$

Let

$$
\Omega(f ; \delta)=\sup _{0<h \leq \delta, x \geq 0} \frac{|f(x+h)-f(x)|}{1+(x+h)^{2}}, \quad \text { for every } f \in C_{x^{2}}^{*}[0, \infty) .
$$

The weighted modulus of continuity $\Omega(f ; \delta)$ was defined by Yuksel and Ispir in [14]. It is well known that $\Omega(f ; \delta)$ has the following properties. 
Lemma 4 ([14]) Let $f \in C_{x^{2}}^{*}[0, \infty)$, then:

(i) $\Omega(f ; \delta)$ is a monotone increasing function of $\delta$.

(ii) For each $f \in C_{x^{2}}^{*}[0, \infty), \lim _{\delta \rightarrow 0^{+}} \Omega(f ; \delta)=0$.

(iii) For each $m \in \mathbb{N} \backslash\{0\}, \Omega(f ; m \delta) \leq m \Omega(f ; \delta)$.

(iv) For each $\lambda \in \mathbb{R}^{+}, \Omega(f ; \lambda \delta) \leq(1+\lambda) \Omega(f ; \delta)$.

Theorem 4 Let $f \in C_{x^{2}}^{*}[0, \infty)$ and $q=q_{n} \in(0,1)$ such that $q_{n} \rightarrow 1$ and $[n]_{q_{n}} \rightarrow \infty$ as $n \rightarrow \infty$, then there exists a positive constant $A$ such that the inequality

$$
\sup _{x \in(0, \infty)} \frac{\left|G_{n, q_{n}}^{\alpha, \beta}(f ; x)-f(x)\right|}{\left(1+x^{2}\right)^{\frac{5}{2}}} \leq A \Omega\left(f ; \frac{1}{\sqrt{[n+2]}]_{q_{n}}}\right)
$$

holds.

Proof For $t>0, x \in(0, \infty)$ and $\delta>0$, by the definition of $\Omega(f ; \delta)$ and Lemma 4, we get

$$
\begin{aligned}
|f(t)-f(x)| & \leq(1+(x+|x-t|))^{2} \Omega(f ;|t-x|) \\
& \leq 2\left(1+x^{2}\right)\left(1+(t-x)^{2}\right)\left(1+\frac{|t-x|}{\delta}\right) \Omega(f ; \delta) .
\end{aligned}
$$

Since $G_{n, q_{n}}^{\alpha, \beta}$ is linear and positive, we have

$$
\begin{aligned}
& \left|G_{n, q_{n}}^{\alpha, \beta}(f ; x)-f(x)\right| \\
& \quad \leq 2\left(1+x^{2}\right) \Omega(f ; \delta)\left\{1+G_{n, q_{n}}^{\alpha, \beta}\left[(t-x)^{2} ; x\right]+G_{n, q_{n}}^{\alpha, \beta}\left[\left(1+(t-x)^{2}\right) \frac{|t-x|}{\delta} ; x\right]\right\} .
\end{aligned}
$$

From Remark 2, we have

$$
G_{n, q_{n}}^{\alpha, \beta}\left((t-x)^{2} ; x\right) \leq A_{1} \frac{1+x^{2}}{[n+2]_{q_{n}}},
$$

for some positive constant $A_{1}$. To estimate the second term of (22), applying the CauchySchwartz inequality, we have

$$
G_{n, q_{n}}^{\alpha, \beta}\left(\left(1+(t-x)^{2}\right) \frac{|t-x|}{\delta} ; x\right) \leq 2\left(G_{n, q_{n}}^{\alpha, \beta}\left(1+(t-x)^{4} ; x\right)\right)^{\frac{1}{2}}\left(G_{n, q_{n}}^{\alpha, \beta}\left(\frac{(t-x)^{2}}{\delta^{2}} ; x\right)\right)^{\frac{1}{2}} .
$$

By Remark 2 and (23), there exist two positive constants $A_{2}, A_{3}$ such that

$$
\left(G_{n, q_{n}}^{\alpha, \beta}\left(1+(t-x)^{4} ; x\right)\right)^{\frac{1}{2}} \leq A_{2}\left(1+x^{2}\right)
$$

and

$$
\left(G_{n, q_{n}}^{\alpha, \beta}\left(\frac{(t-x)^{2}}{\delta^{2}} ; x\right)\right)^{\frac{1}{2}} \leq \frac{A_{3}}{\delta} \sqrt{\frac{1+x^{2}}{[n+2]_{q_{n}}}} .
$$

Now we take $A=1+2 A_{1}+2 A_{2} A_{3}$ and $\delta=\frac{1}{\sqrt{[n+2]_{q_{n}}}}$, and combining the above estimates, we obtain the inequality (21). 


\section{Weighted approximation and Voronovskaya type asymptotic formula}

In this section we will discuss the weighted approximation theorem and Voronovskaya type asymptotic formula.

Theorem 5 Let the sequence $q=\left\{q_{n}\right\}$ satisfy $0<q_{n}<1, q_{n} \rightarrow 1$ and $[n]_{q_{n}} \rightarrow \infty$ as $n \rightarrow \infty$. Then for $f \in C_{x^{2}}^{*}[0, \infty)$, we have

$$
\lim _{n \rightarrow \infty}\left\|G_{n, q_{n}}^{\alpha, \beta}(f)-f\right\|_{x^{2}}=0 .
$$

Proof Using the Korovkin theorem in [15], we know that it is sufficient to verify the following three equations:

$$
\lim _{n \rightarrow \infty}\left\|G_{n, q_{n}}^{\alpha, \beta}\left(t^{k} ; x\right)-x^{k}\right\|_{x^{2}}=0, \quad k=0,1,2 .
$$

Since $G_{n, q_{n}}^{\alpha, \beta}(1 ; x)=1,(28)$ holds true for $k=0$.

By Lemma 2, for $n>1$, we have

$$
\begin{aligned}
\left\|G_{n, q_{n}}^{\alpha, \beta}(t ; x)-x\right\|_{x^{2}} & =\sup _{x \in[0, \infty)}\left\{\left|\left\{\frac{\sqrt{q_{n}}[n]_{q_{n}}[n+1]_{q_{n}}}{\left([n]_{q_{n}}+\beta\right)[n+2]_{q_{n}}}-1\right\} x+\frac{\alpha}{[n]_{q_{n}}+\beta}\right| \times \frac{1}{1+x^{2}}\right\} \\
& \leq\left|\frac{\sqrt{q_{n}}[n]_{q_{n}}[n+1]_{q_{n}}}{\left([n]_{q_{n}}+\beta\right)[n+2]_{q_{n}}}-1\right| \sup _{x \in[0, \infty)} \frac{x}{1+x^{2}}+\frac{\alpha}{[n]_{q_{n}}+\beta} \sup _{x \in[0, \infty)} \frac{1}{1+x^{2}} \\
& \leq\left|\frac{\sqrt{q_{n}}[n]_{q_{n}}[n+1]_{q_{n}}}{\left([n]_{q_{n}}+\beta\right)[n+2]_{q_{n}}}-1\right|+\frac{\alpha}{[n]_{q_{n}}+\beta} \\
& \leq\left|\frac{\sqrt{q_{n}}[n+1]_{q_{n}}}{[n+2]_{q_{n}}}-1\right|+\frac{\beta \sqrt{q_{n}}[n+1]_{q_{n}}}{\left([n]_{q_{n}}+\beta\right)[n+2]_{q_{n}}}+\frac{\alpha}{[n]_{q_{n}}+\beta} \\
& \leq\left|\frac{1}{\sqrt{q_{n}}}-1\right|+\frac{1}{\sqrt{q_{n}}[n+2]_{q_{n}}}+\frac{\alpha+\beta \sqrt{q_{n}}}{[n]_{q_{n}}+\beta} .
\end{aligned}
$$

Thus

$$
\lim _{n \rightarrow \infty}\left\|G_{n, q_{n}}^{\alpha, \beta}(t ; x)-x\right\|_{x^{2}}=0 .
$$

Similarly, for $n>2$, we have

$$
\begin{aligned}
\left\|G_{n, q_{n}}^{\alpha, \beta}\left(t^{2} ; x\right)-x^{2}\right\|_{x^{2}}= & \sup _{x \in[0, \infty)}\left\{\mid\left(\frac{[n]_{q_{n}}}{[n]_{q_{n}}+\beta}\right)^{2} x^{2}+\frac{2 \alpha \sqrt{q_{n}}[n]_{q_{n}}[n+1]_{q_{n}}}{\left([n]_{q_{n}}+\beta\right)^{2}[n+2]_{q_{n}}} x\right. \\
& \left.+\left(\frac{\alpha}{[n]_{q_{n}}+\beta}\right)^{2}-x^{2} \mid \times \frac{1}{1+x^{2}}\right\} \\
\leq & \frac{\beta^{2}}{\left([n]_{q_{n}}+\beta\right)^{2}}+\frac{2 \beta}{[n]_{q_{n}}+\beta}+\frac{\alpha^{2}}{\left([n]_{q_{n}}+\beta\right)^{2}}+\frac{2 \alpha \sqrt{q_{n}}[n]_{q_{n}}[n+1]_{q_{n}}}{\left([n]_{q_{n}}+\beta\right)^{2}[n+2]_{q_{n}}} \\
\leq & \frac{\alpha^{2}+\beta^{2}}{\left([n]_{q_{n}}+\beta\right)^{2}}+\frac{2 \alpha \sqrt{q_{n}}+2 \beta}{[n]_{q_{n}}+\beta}
\end{aligned}
$$

which implies that

$$
\lim _{n \rightarrow \infty}\left\|G_{n, q_{n}}^{\alpha, \beta}\left(t^{2} ; x\right)-x^{2}\right\|_{x^{2}}=0 .
$$

The proof is completed. 
Finally, we give a Voronovskaya type asymptotic formula for $G_{n, q_{n}}^{\alpha, \beta}(f ; x)$ by means of the second and the fourth central moments.

Theorem 6 Let $f$ be a bounded and integrable function on the interval $(0, \infty)$ and $\left\{q_{n}\right\}_{n=1}^{\infty}$ be a sequence such that $0<q_{n}<1$ and $q_{n} \rightarrow 1$ as $n \rightarrow \infty$. Suppose that the second derivative $f^{\prime \prime}(x)$ exists at a point $x \in(0, \infty)$, then we have

$$
\lim _{n \rightarrow \infty}[n+2]_{q_{n}}\left(G_{n, q_{n}}^{\alpha, \beta}(f ; x)-f(x)\right)=(\alpha-(1+\beta) x) f^{\prime}(x)+x^{2} f^{\prime \prime}(x) .
$$

Proof By the Taylor formula we have

$$
f(t)-f(x)=(t-x) f^{\prime}(x)+\frac{1}{2} f^{\prime \prime}(x)(t-x)^{2}+r(t, x)(t-x)^{2},
$$

where $r(t, x)$ is bounded and $\lim _{t \rightarrow x} r(t, x)=0$. By applying the operator $G_{n, q}^{\alpha, \beta}(f ; x)$ to the above equation we obtain

$$
\begin{aligned}
G_{n, q_{n}}^{\alpha, \beta}(f ; x)-f(x)= & f^{\prime}(x) G_{n, q_{n}}^{\alpha, \beta}((t-x) ; x)+\frac{1}{2} f^{\prime \prime}(x) G_{n, q_{n}}^{\alpha, \beta}\left((t-x)^{2} ; x\right) \\
& +G_{n, q_{n}}^{\alpha, \beta}\left(r(t, x)(t-x)^{2} ; x\right) \\
= & f^{\prime}(x) A_{n, q_{n}}^{\alpha, \beta}(x)+\frac{1}{2} f^{\prime \prime}(x) B_{n, q_{n}}^{\alpha, \beta}(x)+G_{n, q_{n}}^{\alpha, \beta}\left(r(t, x)(t-x)^{2} ; x\right) .
\end{aligned}
$$

By direct calculation, we obtain

$$
\begin{aligned}
{[n+2]_{q_{n}} A_{n, q_{n}}^{\alpha, \beta}(x) } & =\left\{\frac{\sqrt{q_{n}}[n]_{q_{n}}[n+1]_{q_{n}}}{\left([n]_{q_{n}}+\beta\right)}-[n+2]_{q_{n}}\right\} x+\frac{\alpha[n+2]_{q_{n}}}{[n]_{q_{n}}+\beta} \\
& =\left\{\sqrt{q_{n}}[n+1]_{q_{n}}-[n+2]_{q_{n}}\right\} x-\left\{\frac{\sqrt{q_{n}} \beta[n+1]_{q_{n}}}{[n]_{q_{n}}+\beta}\right\} x+\frac{\alpha[n+2]_{q_{n}}}{[n]_{q_{n}}+\beta} \\
& =\left(\sqrt{q_{n}}-q_{n}\right)[n+1]_{q_{n}} x-\left\{1+\frac{\sqrt{q_{n}} \beta[n+1]_{q_{n}}}{[n]_{q_{n}}+\beta}\right\} x+\frac{\alpha[n+2]_{q_{n}}}{[n]_{q_{n}}+\beta} \\
& =\frac{\sqrt{q_{n}}\left(1-q_{n}^{n+1}\right)}{1+\sqrt{q_{n}}} x-\left\{1+\frac{\sqrt{q_{n}} \beta[n+1]_{q_{n}}}{[n]_{q_{n}}+\beta}\right\} x+\frac{\alpha[n+2]_{q_{n}}}{[n]_{q_{n}}+\beta} \\
& \rightarrow \alpha-(1+\beta) x \quad(n \rightarrow \infty) .
\end{aligned}
$$

Similarly,

$$
\begin{aligned}
{[n} & +2]_{q_{n}}\left[\left(\frac{[n]_{q_{n}}}{[n]_{q_{n}}+\beta}\right)^{2}-\frac{2 \sqrt{q_{n}}[n]_{q_{n}}[n]_{q_{n}}}{\left([n]_{q_{n}}+\beta\right)\left([n+2]_{q_{n}}\right)}+1\right] \\
& =2\left\{[n+2]_{q_{n}}-\frac{\sqrt{q_{n}}[n]_{q_{n}}[n+1]_{q_{n}}}{[n]_{q_{n}}+\beta}-\frac{\beta[n+2]_{q_{n}}}{[n]_{q_{n}}+\beta}\right\} \\
& =2\left\{[n+2]_{q_{n}}-\sqrt{q_{n}}[n+1]_{q_{n}}+\frac{\sqrt{q_{n}} \beta[n+1]_{q_{n}}}{[n]_{q_{n}}+\beta}-\frac{\beta[n+2]_{q_{n}}}{[n]_{q_{n}}+\beta}\right\} \\
& =2\left\{1+\left(q_{n}-\sqrt{q_{n}}\right)[n+1]_{q_{n}}+\frac{\sqrt{q_{n}} \beta[n+1]_{q_{n}}}{[n]_{q_{n}}+\beta}-\frac{\beta[n+2]_{q_{n}}}{[n]_{q_{n}}+\beta}\right\} \\
& \rightarrow 2 \quad(n \rightarrow \infty) .
\end{aligned}
$$


That means

$$
[n+2]_{q_{n}} B_{n, q_{n}}^{\alpha, \beta}(x) \rightarrow 2 x^{2} \quad(n \rightarrow \infty) .
$$

On the other hand, by simple calculation we obtain

$$
\begin{aligned}
& {[n+2]_{q_{n}}\left(\frac{[n+3]_{q_{n}}[n+4]_{q_{n}}}{[n]_{q_{n}}[n-1]_{q_{n}}}-1\right) \rightarrow 8 \quad(n \rightarrow \infty),} \\
& {[n+2]_{q_{n}}\left(\frac{[n+3]_{q_{n}}}{[n]_{q_{n}}}-1\right) \rightarrow 3 \quad(n \rightarrow \infty) .}
\end{aligned}
$$

Thus from Remark 2, we have

$$
[n+2]_{q_{n}} G_{n, q_{n}}^{\alpha, \beta}\left((t-x)^{4} ; x\right) \rightarrow 0 \quad(n \rightarrow \infty) .
$$

Since $r(t, x)$ is bounded and $\lim _{t \rightarrow x} r(t, x)=0$, then for any given $\epsilon>0$, there exists a $\delta>0$ such that

$$
|r(t, x)| \leq \epsilon+\frac{M}{\delta^{2}}(t-x)^{2} \quad(t, x \in(0, \infty), M \text { is a positive constant }) .
$$

Thus

$$
\begin{aligned}
& {[n+2]_{q_{n}}\left|G_{n, q_{n}}^{\alpha, \beta}\left(r(t, x)(t-x)^{2} ; x\right)\right|} \\
& \quad \leq \epsilon[n+2]_{q_{n}} G_{n, q_{n}}^{\alpha, \beta}\left((t-x)^{2} ; x\right)+\frac{M}{\delta^{2}}[n+2]_{q_{n}} G_{n, q_{n}}^{\alpha, \beta}\left((t-x)^{4} ; x\right) \rightarrow 0 \quad(n \rightarrow \infty) .
\end{aligned}
$$

The proof is completed.

\section{Competing interests}

The authors declare that they have no competing interests.

\section{Authors' contributions}

All authors contributed equally to the writing of this paper. All authors read and approved the final manuscript.

\section{Acknowledgements}

This work is supported by the National Natural Science Foundation of China (Grant No. 61170324 and Grant No. 61100105). The authors thank the associate editor and the referees for their important comments and suggestions, which improved the quality of the paper.

Received: 25 October 2013 Accepted: 11 February 2014 Published: 25 Feb 2014

\section{References}

1. Karsli, $\mathrm{H}$ : Rate of convergence of a new Gamma type operators for functions with derivatives of bounded variation Math. Comput. Model. 45(5-6), 617-624 (2007)

2. Karsli, H, Gupta, $V$, Izgi, A: Rate of point wise convergence of a new kind of gamma operators for functions of bounded variation. Appl. Math. Lett. 22, 505-510 (2009)

3. Kim, T: q-Generalized Euler numbers and polynomials. Russ. J. Math. Phys. 13(3), 293-298 (2006)

4. Kim, T: Some identities on the $q$-integral representation of the product of several $q$-Bernstein-type polynomial. Abstr. Appl. Anal. 2011, Article ID 634675 (2011)

5. Gasper, G, Rahman, M: Basic Hypergeometrik Series. Encyclopedia of Mathematics and Its Application, vol. 35. Cambridge University Press, Cambridge (1990)

6. Kac, VG, Cheung, P: Quantum Calculus. Universitext. Springer, New York (2002)

7. Aral, A, Gupta, V, Agarwal, RP: Applications of q-Calculus in Operator Theory. XII. Springer, New York (2013). doi:10.1007/978-1-4614-6946-9

8. Jackson, FH: On a q-definite integrals. Q. J. Pure Appl. Math. 41, 193-203 (1910) 
9. Koornwinder, TH: $q$-Special functions, a tutorial. In: Gerstenhaber, M, Stasheff, J (eds.) Deformation Theory and Quantum Groups with Applications to Mathematical Physics. Contemp. Math., vol. 134. Am. Math. Soc., Providence (1992)

10. De Sole, A, Kac, VG: On integral representations of q-gamma and q-beta functions. Atti Accad. Naz. Lincei, Rend. Lincei, Mat. Appl. 9(16), 11-29 (2005)

11. Cai, Q, Zeng, XM: On the convergence of a kind of q-gamma operators. J. Inequal. Appl. 2013, 105 (2013)

12. Gupta, V, Agarwal, RP: Convergence Estimates in Approximation Theory. VIII. Springer, New York (2014)

13. DeVore, RA, Lorentz, GG: Constructive Approximation. Springer, Berlin (1993)

14. Yuksel, I, Ispir, N: Weighted approximation by a certain family of summation integral-type operators. Comput. Math. Appl. 52(10-11), 1463-1470 (2006)

15. Gadjiev, AD: Theorems of the type of P.P. Korovkin type theorems. Mat. Zametki 20(5), 781-786 (1976) (English translation: Math. Notes 20(5-6), 996-998 (1976))

10.1186/1029-242X-2014-94

Cite this article as: Zhao et al.: Some approximation properties of a kind of $q$-Gamma-Stancu operators. Journal of Inequalities and Applications 2014, 2014:94

\section{Submit your manuscript to a SpringerOpen ${ }^{\circ}$ journal and benefit from:}

- Convenient online submission

- Rigorous peer review

Immediate publication on acceptance

- Open access: articles freely available online

- High visibility within the field

- Retaining the copyright to your article 